\title{
Immune-related Adverse Events Associated with Cancer Immunotherapy: A Review for the Practicing Rheumatologist
}

\author{
Shahin Jamal, Marie Hudson, Aurore Fifi-Mah (1D, and Carrie Ye
}

\begin{abstract}
Immune checkpoint inhibitors have revolutionized cancer therapy by blocking inhibitory pathways of the immune system to fight cancer cells. Their use is often limited by the development of autoimmune toxicities, which can affect multiple organ systems and are referred to as immune-related adverse events (irAE). Among these are rheumatologic irAE, including inflammatory arthritis, myositis, vasculitis, and others. Rheumatologic irAE seem to be different from irAE in other organs and from traditional autoimmune diseases in that they can occur early or have delayed onset, and can persist chronically, even after cancer therapy is terminated. Because immune checkpoint inhibitors are increasingly used for many types of cancer, it is important for oncologists and rheumatologists to recognize and manage toxicities early. In this review, we discuss currently approved immune checkpoint inhibitors and their mechanisms of action and systemic toxicities, with a focus on the management and effect on further cancer therapy of rheumatic irAE. (First Release December 15 2019; J Rheumatol 2020;47:166-75; doi:10.3899/jrheum.190084)
\end{abstract}

Key Indexing Terms:

IMMUNE CHECKPOINT INHIBITORS INFLAMMATORY ARTHRITIS

Immunotherapy has emerged as a new pillar in the treatment of cancer and has transformed outcomes of patients with previously untreatable malignancies ${ }^{1,2}$. Unlike traditional chemotherapy, which commonly has the secondary effect of immunosuppression, modern immunotherapy aims at upregulating the immune system to augment antitumor responses. Immune checkpoint inhibitors (ICI) have emerged as one of the most promising forms of immunotherapy ${ }^{3}$.

ICI increase antitumor activity by blocking intrinsic downregulators of the immune system, including $\mathrm{T}$ cell lymphocyte costimulation inhibitor (CTLA-4), programmed death protein-1 (PD-1), and programmed death ligand-1

From the Division of Rheumatology, Vancouver General Hospital, and Department of Medicine, University of British Columbia, Vancouver, British Columbia; Division of Rheumatology, Jewish General Hospital and Lady Davis Institute; Department of Medicine, McGill University, Montreal, Quebec; Division of Rheumatology, University of Calgary, Calgary; Division of Rheumatology, Department of Medicine, University of Alberta, Edmonton, Alberta, Canada.

S. Jamal, MD, MSc, Division of Rheumatology, Vancouver General Hospital, and Department of Medicine, University of British Columbia; M. Hudson, MD, MPH, Division of Rheumatology, Jewish General Hospital and Lady Davis Institute; A. Fifi-Mah, MD, Division of Rheumatology, University of Calgary; C. Ye, MD, Division of Rheumatology, Department of Medicine, University of Alberta .

Address correspondence to Dr. S. Jamal, 2775 Laurel Ave., Suite 8205B, Vancouver, British Columbia V5Z 1M9, Canada. E-mail:Shahin.jamal@vch.ca

Accepted for publication June 28, 2019.

\section{ADVERSE EVENTS MYOSITIS}

\section{IMMUNOTHERAPY VASCULITIS}

$(\mathrm{PD}-\mathrm{L} 1)^{4}$. Normally, these regulatory receptors, or checkpoints, maintain the balance between $\mathrm{T}$ cell activation and inhibition. The primary aim of ICI is to reduce the suppression of effector T cells, particularly CD8+ T cells, improving their ability to mount tumor-specific immune responses $^{5}$. CTLA-4 is upregulated early after $\mathrm{T}$ cell activation in central tissues including lymphoid and thymic tissues (Figure 1). It transmits an inhibitory signal to activated $\mathrm{T}$ cells at a proximal step in the immune response, by preferentially binding to $\mathrm{CD} 80 / 86$ expressed by antigen presenting cells (APC), thereby blocking the second signal required for $\mathrm{T}$ cell activation. Antibodies targeting CTLA- 4 neutralize this central checkpoint, allowing ongoing $\mathrm{T}$ cell activation and thereby enhancing their anti-tumor activity.

In contrast, PD-1 is believed to play a role in T cell inhibition in the peripheral tissues, at a later stage of the immune response (Figure 1). When effector T cells encounter continuous antigen (as in chronic infection or cancer), they lose the ability to respond to the antigen - a condition known as $\mathrm{T}$ cell exhaustion. This is partly mediated by the enhanced expression of immune checkpoints such as PD-1. The binding of PD-1 to its ligands, PD-L1 and PD-L2 (which are expressed by many tumor cells), interferes with downstream signaling and can lead to $\mathrm{T}$ cell exhaustion. Antibodies that block the interaction of PD-1 with PD-L1/PD-L2 serve to neutralize this checkpoint, restoring $\mathrm{T}$ cell effector function ${ }^{5,6}$. 
CTLA-4 mediates inhibition in the central lymphoid compartment.

CTLA-4 modulates the immune response by:

- Preferentially binding CD80/86 proteins on APCs,

- Preventing the binding of CD28 (2nd signal needed for T cell activation), and

- Inhibiting T cell activation.

Antibodies that block CTLA-4 can lead to ongoing T cell activation. These $T$ cells can then migrate to the peripheral tissues and attack tumor cells.

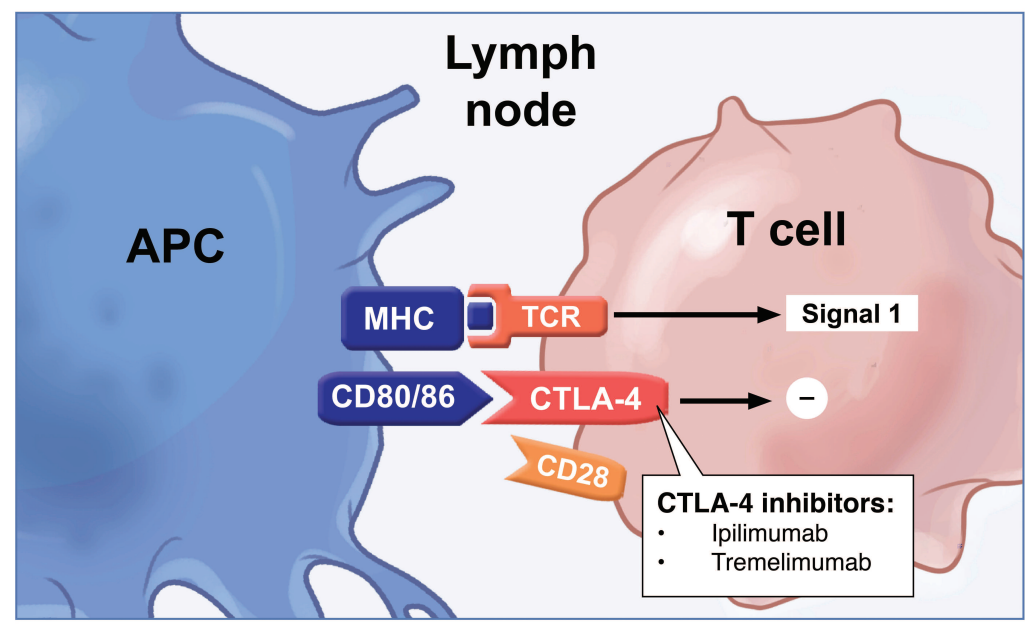

Abbreviations: APC, antigen-presenting cell; CD28, cluster of differentiation 28

CD80/86, cluster of differentiation 80 or 86 ; CTLA-4, cytotoxic T-lymphocyte-associated antigen 4; MHC, major histocompatibility complex; TCR, T cell receptor.

PD-1 mainly exerts its inhibitory effect on T cells in peripheral tissues.

The binding of PD-1 on T cells to PD-L1/PD-L2 on tumor cells can lead to:

- Inhibition of downstream signalling,

- Suppression of T cell function, and

- T cell exhaustion.

Antibodies that block PD-1, PD-L1, and PD-L2 can restore T cell effector function for reactivated anti-tumor response.

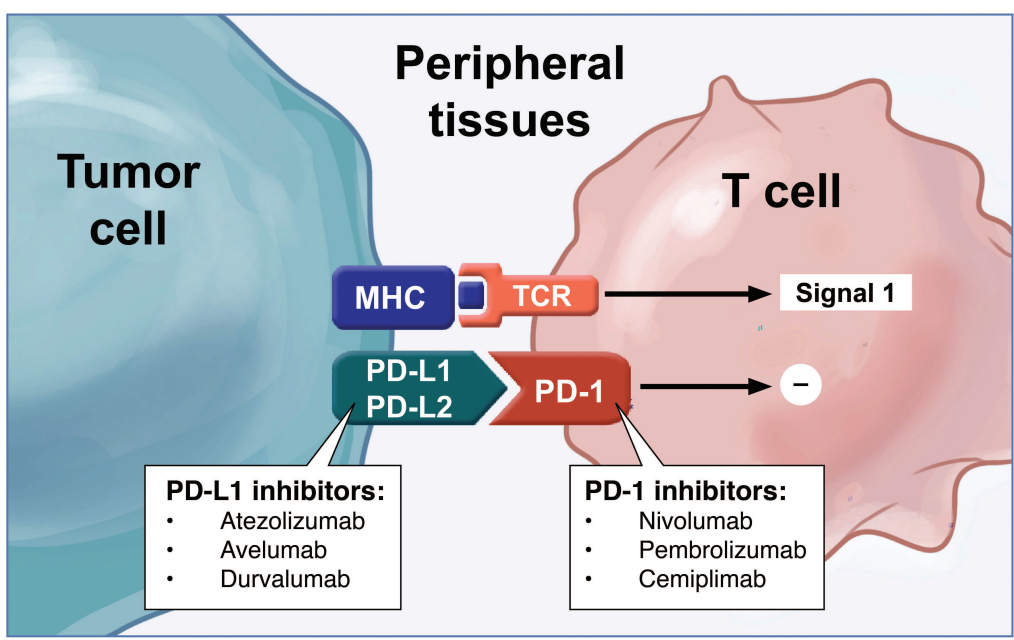

Abbreviations: MHC, major histocompatibility complex; PD-1, programmed death 1;

PD-L1/2, programmed death-ligand 1 or 2 ; TCR, T cell receptor.

Figure 1. Mechanism of action of immune checkpoint inhibitors. 
The widespread use of ICI has led to remarkable clinical outcomes, with complete remissions and sustained clinical responses seen in some patients with previously refractory cancers such as melanoma, lung cancer, renal cell carcinoma, and Hodgkin lymphoma. To date, the US Food and Drug Administration and Health Canada have approved 7 ICI (and 1 combination), which target either CTLA-4 or PD-1/PD-L1 pathways for a variety of different cancers (Table 1$)^{7}$. Indications for these (either alone or in combination) continue to grow rapidly as more data become available. Other immune checkpoint pathways have also been identified and are currently being investigated for clinical use in an increasing number of cancer types and stages.

\section{Immune-related adverse events associated with ICI}

Unfortunately, activating the immune system to fight cancer can also lead to serious, undesirable off-target immune and inflammatory events known as immune-related adverse events (irAE). These have been reported in up to $80 \%$ of patients receiving monotherapy and up to $95 \%$ with combination therapy (PD-1 and CTLA-4) $)^{8,9,10,11,12}$. There have been higher rates reported with CTLA-4 blockade than with PD-1 blockade ${ }^{13}$. The clinical spectrum of irAE is broad and can affect multiple organ systems (Figure 2). Further, many patients can experience more than 1 irAE involving multiple organ systems ${ }^{11}$. The most common irAE include rash, colitis, thyroiditis, hypophysitis, hepatitis, pneumonitis, and arthritis, with clinical severity ranging from mild to severe and occasionally fatal. Most events occur within the first 3-4 months of therapy but can occur any time during treatment and even years after cessation of immunotherapy. Although some irAE can be transient, others require chronic immunosuppression. In addition to the morbidity of irAE, a recent metaanalysis reported a fatality rate of $1.3 \%$ and as many as a third of patients are forced to stop these potentially life-saving therapies because of toxicity ${ }^{14}$. Early symptoms are often nonspecific, including fatigue and malaise; further, many patients with cancer assume these side effects are expected and often underreport symptoms, leading to delay in diagnosis. Therefore, awareness and early recognition is essential to optimize longterm outcomes.

Rheumatic irAE. Rheumatic irAE (Rh-irAE) secondary to immunotherapy are likely underreported in clinical trials with the majority of our knowledge coming from case reports and case series ${ }^{15}$. Thus, our understanding of the prevalence, incidence, clinical features, optimal management, and prognosis of Rh-irAE continues to evolve with ongoing clinical experience. In early clinical trials, arthralgias and myalgias were the most commonly reported Rh-irAE, with a prevalence of $1-43 \%$ and $2-20 \%$, respectively ${ }^{16}$. With the increased use of ICI in clinical practice, a wide range of Rh-irAE have been described, most commonly inflammatory arthritis, but also myositis, vasculitis, new-onset fractures, resorptive bone lesions, sicca syndrome, and sarcoidosis ${ }^{17,18,19}$. Inflammatory arthritis. Inflammatory arthritis secondary to ICI (ir-IA) has been described in several case series and retrospective cohort studies $6,17,18,20-24$. The prevalence was reported as $3.8 \%$ in one study ${ }^{18}$. The most common patterns include (1) polymyalgia rheumatica (PMR)-like (shoulder and pelvic girdle stiffness), (2) small joint symmetric inflammatory arthritis (predominantly hand) with diffuse tenosynovitis, and (3) large joint, asymmetric oligoarthritis,

Table 1. List of FDA and Health Canada-approved immune checkpoint inhibitors and their indications.

\begin{tabular}{|c|c|c|}
\hline Name & Molecular Target & Indications \\
\hline Ipilimumab & CTLA-4 & Metastatic melanoma \\
\hline Nivolumab & PD-1 & $\begin{array}{l}\text { Metastatic melanoma, NSCLC (squamous and n } \\
\text { nonsquamous), renal cell carcinoma, Hodgkin lymphoma, } \\
\text { SCCHN, urothelial carcinoma, HCC, dMMR and MSI-H } \\
\text { colorectal cancer }\end{array}$ \\
\hline Pembrolizumab & PD-1 & $\begin{array}{l}\text { Metastatic melanoma, Hodgkin lymphoma, NSCLC, } \\
\text { urothelial carcinoma, HNSCC, dMMR of MSI-H solid } \\
\text { tumor, PD-L1 gastric and gastroesophageal junction } \\
\text { adenocarcinoma }\end{array}$ \\
\hline Atezolizumab & PD-L1 & Metastatic urothelial carcinoma, metastatic NSCLC \\
\hline Avelumab & PD-L1 & Metastatic merkel cell carcinoma, urothelial carcinoma \\
\hline Durvalumab & PD-L1 & Urothelial carcinoma, NSCLC \\
\hline Cemiplimab & PD-1 & Squamous cell skin carcinoma \\
\hline $\begin{array}{l}\text { Combination ipilimumab + } \\
\text { nivolumab }\end{array}$ & CTLA-4 + PD-1 & $\begin{array}{l}\text { Metastatic melanoma, RCC, HNSCC, metastatic NSCLC } \\
\text { with high PD-L1 expression }\end{array}$ \\
\hline
\end{tabular}

FDA: US Food and Drug Administration; CTLA-4: T cell lymphocyte costimulation inhibitor; PD-1: programmed death protein-1; PD-L1: programmed death ligand-1; NSCLC: non small-cell lung carcinoma; RCC: renal cell carcinoma; SCCHN: squamous cell carcinoma of the head and neck; HCC: hepatocellular carcinoma; dMMR: mismatch repair deficient; MSI-H: microsatellite instability-high; HNSCC: head and neck squamous cell carcinoma. 


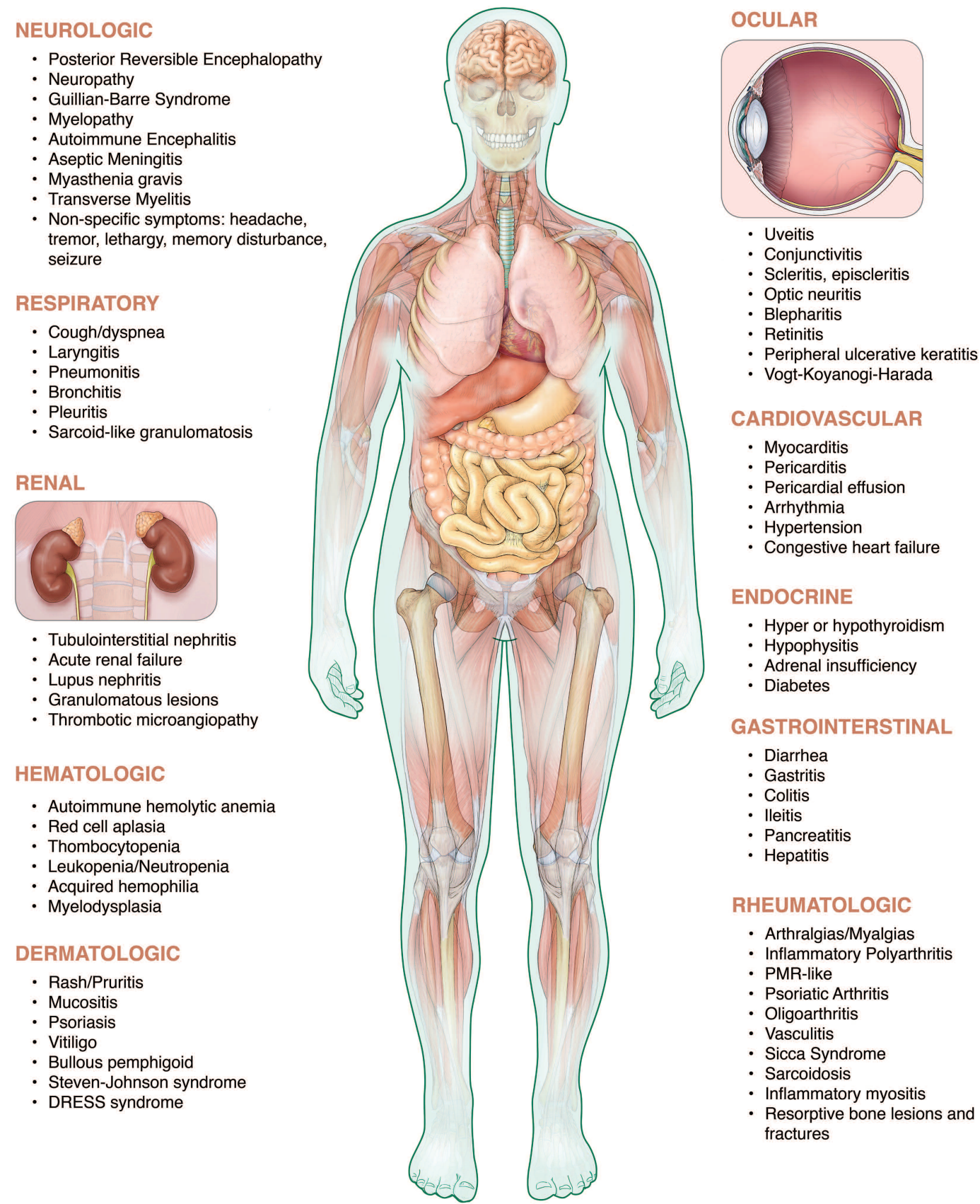

Figure 2. Systemic irAE associated with immune checkpoint inhibitors. irAE: immune-related adverse events; PMR: polymyalgia rheumatica; DRESS syndrome: Drug Rash with Eosinophilia and Systemic Symptoms syndrome.

predominantly involving the knees. In addition, there have been reported cases of a reactive arthritis-like triad (urethritis, conjunctivitis, and arthritis) ${ }^{17}$, and psoriatic arthritis.

Ir-IA may be different from the classic inflammatory rheumatic diseases that they mimic and may have differing longterm outcomes. There has been no predilection by sex reported, with generally equal cases in men and women. The majority of cases have been seronegative [although rheumatoid factor (RF)-positive and cyclic citrullinated peptide antibodies (CCP)-positive cases have been reported], with high prevalence of enthesitis/tenosynovitis that often requires higher doses of corticosteroids than traditionally used. Although ir-IA have been reported after 1 dose, they can occur up to 2 years after immunotherapy and can often become chronic, despite stopping immunotherapy, unlike other irAE such as colitis and pneumonitis. Joint involvement pattern at baseline, severity of symptom onset, and requirement of longterm immunosuppression may differ by the underlying immunotherapy regimen ${ }^{25}$.

In one case series of 26 patients of European descent with

$$
\text { Personal non-commercial use only. The Journal of Rheumatology Copyright @ } 2020 \text {. All rights reserved. }
$$


ir-IA, researchers found that there was a higher prevalence of shared epitope (SE) alleles and DRB1*04:05 compared to population controls, and similar prevalence of at least 1 SE allele compared to rheumatoid arthritis patients of European descent. Interestingly, despite being SE-positive, these patients were negative for RF and CCP antibody, which raises questions regarding potential underlying mechanisms. Further, they found certain clinical features (enthesitis, trigger fingers, inflammatory low back pain, reactive arthritis) only in patients lacking SE alleles. Research is needed to determine whether distinct HLA alleles are associated with unique ir-IA phenotypes, and their risk of development ${ }^{26}$.

In addition to inflammatory arthritis (presenting with synovitis), there is emerging literature on a subset of patients with inflammatory-type joint pain without synovitis ${ }^{6,22}$. In our experience ${ }^{24}$, similar to that reported in the literature, these patients can present with debilitating pain and progressive osteophytosis, which responds to low-dose corticosteroid and antimalarial therapy.

Myositis. Myositis is an increasingly recognized irAE (ir-Myositis), although it remains relatively uncommon, affecting $<1 \%$ of patients exposed to $\mathrm{ICI}^{27,28}$. Ir-Myositis offers some unique insights into traditional autoimmune myositides (AIM). First, if ICI are considered as a class, this "single" exposure can recapitulate a broad spectrum of AIM, including dermatomyositis ${ }^{29,30,31}$, necrotizing myositis ${ }^{27,32}$, and granulomatous myositis ${ }^{33}$. This supports the observation that the same exposure could be responsible for more than 1 traditional AIM. On the other hand, ir-Myositis has been reported to overlap with myocarditis and myasthenia gravis $^{34,35}$ much more frequently than when observed in the setting of traditional AIM $^{36}$. Similarly, although myositis has been reported to occur in $5 \%$ of patients with thymomas, in one series of 8 patients with thymoma treated with avelumab, $4(50 \%)$ developed myositis ${ }^{37}$. Finally, most (though not all $)^{27,31}$ cases of ir-Myositis from ICI reported to date have been seronegative ${ }^{37,38,39}$. Thus, myositis from irAE probably has important differences from traditional AIM and a subset of ir-Myositis may represent a new entity.

In one of the most detailed series to date $(n=10)$, Touat, et al described a unique constellation of features that characterize ir-Myositis as follows: (1) early (within 2 mos of ICI initiation) and severe onset of symptoms; (2) limb-girdle weakness associated with myalgias, as well as axial and oculomotor weakness; (3) striking creatinine kinase elevations with myopathic changes on electromyography; (4) absence of myositis-specific and antiacetylcholine receptor antibodies; (5) necrosis and inflammation on histopathology; and (6) good response to ICI discontinuation with or without corticosteroids ${ }^{39}$.

Ir-Myositis tends to occur early, often after the first or second dose of ICI. Ir-Myositis ranges from mild, with some cases resolving spontaneously upon discontinuation of the offending drug ${ }^{39}$, to severe peripheral and respiratory weakness requiring mechanical ventilation ${ }^{40}$. Although myositis represents one of the less common irAE, it is associated with a high case fatality rate (17\%), second only to myocarditis $(39.7 \%)^{14}$. Mortality in cases of ir-Myositis overlapping with myocarditis or other neuromuscular symptoms is even higher (over $50 \%$ in one study) ${ }^{41}$, and results from cardiac or respiratory failure 30,39 .

Recognizing myositis from ICI is, in most cases, straightforward, with patients generally presenting with rapidly progressing proximal muscle weakness and elevated muscle enzymes. Other symptoms include distal, axial, and oculobulbar weakness; dysphagia, diaphragmatic weakness, and $\operatorname{rash}^{27,39,42}$. However, diagnosis may be delayed, with some patients attributing their symptoms to their cancer and related problems. Further, ir-Myositis may be difficult to distinguish from cancer-associated myositis, myocarditis, and myasthenia gravis. Finally, elevated liver enzymes have been mistakenly attributed to hepatitis, which is a more common irAE than myositis $^{43}$. In general, a high level of clinical suspicion is essential given the high risk of mortality, and muscle biopsy may be required.

Vasculitis. Cases of vasculitic irAE (ir-Vasculitis) affecting the large-, medium-, or small-size vessels have been reported. A large pharmacovigilance study and a systematic review published in 2018 identified numerous cases of vasculitis ${ }^{44,45}$. Most frequent were large-vessel vasculitis, including giant cell arteritis (GCA) and isolated aortitis, and nervous system vasculitis, including primary angiitis of the central nervous system and peripheral nerve involvement. The median duration of ICI therapy preceding symptom onset was 3 months, ranging from 1 week to 18 months after initial ICI exposure. Several other cases of vasculitis have been described, including 2 cases of acral vasculitis causing digital necrosis with amputation of the distal digits despite aggressive immunosuppression ${ }^{46}$. Proteinase 3-antineutrophil cytoplasmic antibodies granulomatosis with polyangiitis (GPA) was described in a patient following sequential treatment with ipilimumab, then pembrolizumab ${ }^{47}$. Single-organ vasculitis of the uterus ${ }^{48}$, eye ${ }^{49,50}$, and skin $^{51}$ have also been reported. Interestingly, a patient with eosinophilic GPA was successfully treated with ipilimumab, then pembrolizumab for metastatic melanoma without a vasculitis flare ${ }^{52}$

There seems to be little difference between the clinical, biological, or histopathological presentation of ir-Vasculitis and idiopathic forms of these diseases. The predominance of large-vessel vasculitis also fits with the PD-1/PD-L1 pathway dysfunction in GCA highlighted by the work of Zhang, et $a l^{53}$. They showed that the tissue microenvironment of GCA lacks the inhibitory ligand PD-L1 and enriches for PD-1 expressing $\mathrm{T}$ cells. Inhibiting the PD-1/PD-L1 interaction intensified $\mathrm{T}$ cell accumulation in the vessel wall and profoundly enhanced tissue inflammation and remodeling of

Personal non-commercial use only. The Journal of Rheumatology Copyright $\subset$ 2020. All rights reserved 
the vessel wall, affecting the process of neoangiogenesis and intimal hyperplasia. ICI iatrogenically produce a similar effect and could therefore accelerate the autoimmune process in the GCA microenvironment.

\section{General principles for management of irAE}

The Common Terminology Criteria for Adverse Events (CTCAE) can be used to grade the severity of AE and help guide management decisions (Table 2) ${ }^{54}$. Grades 1 and 2 are considered mild, grades 3 and 4 are severe, and grade 5 indicates that the patient died of an AE. In the past few years, multiple groups including the Society for Immunotherapy of Cancer (SITC) Toxicity Management Working Group, the European Society of Medical Oncology (ESMO), and the American Society of Clinical Oncology (ASCO) have released clinical guidelines on management of irAE secondary to immunotherapy, based on expert consensus ${ }^{10,55,56,57,58}$. The goal of treatment is to carefully balance immune function by administering enough immunosuppression to treat $\mathrm{AE}$ and prevent organ damage, without mitigating the antitumor effects of immunotherapy.

Successful management of patients with irAE requires a multidisciplinary, patient-centered approach. Champiat, et al proposed a practical approach to management of patients receiving immunotherapy including prevention, anticipation, detection, treatment, and ongoing monitoring ${ }^{10}$.

Referral to rheumatology. Because delay in diagnosis and treatment of Rh-irAE can lead to longterm disability and disease chronicity, it is important to identify those at risk and refer them early to rheumatology. SITC guidelines have recommended rheumatology referral for any patient with (1) joint swelling, (2) CTCAE grade 2 and above symptoms, (3) symptoms persisting for more than 6 weeks or requiring prednisone $20 \mathrm{mg}$ daily or equivalent that cannot be tapered to $<10 \mathrm{mg} /$ day within 4 weeks, and (4) suspected myositis, presenting with muscle weakness and elevated creatinine.

The decision regarding earlier rheumatology referral depends on geographic variation, oncologist comfort and

Table 2. Common Terminology Criteria for Adverse Events grades (v. 5).

\begin{tabular}{ll}
\hline Grade & Severity of Adverse Event \\
\hline Grade 1 & $\begin{array}{l}\text { Mild; asymptomatic or mild symptoms; clinical or diagnostic } \\
\text { observations only; intervention not indicated } \\
\text { Moderate; minimal, local or noninvasive intervention indicated; } \\
\text { Grade 2 }\end{array}$ \\
Grade 3 & $\begin{array}{l}\text { Severe or medically significant but not immediately } \\
\text { life-threatening; hospitalization or prolongation of } \\
\text { hospitalization indicated; disabling; limiting self-care ADL** }\end{array}$ \\
Grade 4 & $\begin{array}{l}\text { Life-threatening consequences; urgent intervention indicated } \\
\text { Death related to adverse events }\end{array}$ \\
\hline
\end{tabular}

* Instrumental ADL (activities of daily living) refers to preparing meals, shopping, managing money, etc. ** Self-care ADL refers to bathing, dressing, feeding, toileting, etc. experience with rheumatic disease, and availability of local rheumatologists. Ideally, we recommend referral to rheumatology for patients with any rheumatic symptoms (Grade 1 or higher) prior to starting prednisone. We encourage oncologists to develop referral relationships with 1 or 2 local rheumatologists. This will facilitate urgent evaluation of patients and allow the rheumatologist to accrue clinical experience with immunotherapy-related toxicity, leading to improved patient care.

Management of patients with Rh-irAE. The role of the rheumatologist is to diagnose and optimize management of $\mathrm{Rh}$-irAE to facilitate ongoing immunotherapy, if indicated, for the underlying cancer.

It is important for rheumatologists to remember that generalized musculoskeletal symptoms (myalgia, arthralgia, weakness) can be associated with underlying cancer, preexisting arthritis, metastatic disease, infection, or side effects of other medications. They can also occur with other irAE including endocrine, gastrointestinal, and neurological types. Therefore, evaluation of patients requires a careful history and physical examination and familiarity with possible AE involving various systems and organs. Inflammatory markers including C-reactive protein are often helpful biomarkers for diagnosis and monitoring response to therapy, but can be elevated because of underlying malignancy or other nonrheumatic irAE. Radiographs can be helpful to evaluate for erosive disease and exclude metastases. Laboratory tests, biopsies, and autoimmune serologies are recommended as directed by the clinical phenotype. In those with ir-Myositis, careful evaluation for myocarditis and myasthenia gravis should be undertaken.

Current management guidelines released by ASCO, ESMO, and SITC recommend treatment based on severity of clinical presentation. There are currently no clinical trials of optimal management, and the majority of recommendations are based on expert consensus. For those with mild symptoms (Grade 1), conservative management with analgesics and nonsteroidal antiinflammatory drugs is recommended without interruption of immunotherapy. For large joint involvement, intraarticular corticosteroid injection may be beneficial ${ }^{59}$.

For Grade 2 reactions, oral corticosteroids are recommended $(0.5-1.0 \mathrm{mg} / \mathrm{kg} / \mathrm{d})$, tapering over 4-6 weeks, while holding ICI therapy. If prednisone taper is not tolerated, disease-modifying antirheumatic drugs (DMARD) should be started, depending on clinical presentation. In our experience, some patients with ir-IA have a good response to low-dose (10-20 mg) prednisone and can tolerate ongoing ICI therapy, particularly with concurrent DMARD therapy.

For Grade 3 or above reactions, high-dose oral or intravenous corticosteroids are recommended, with permanent discontinuation of ICI. Patients should be monitored closely, either in hospital or with close outpatient followup, until resolution of symptoms. Early use of concomitant steroid-sparing agents may be needed, depending on clinical

Personal non-commercial use only. The Journal of Rheumatology Copyright (c) 2020. All rights reserved. 
presentation and response to corticosteroids. The choice of agent usually depends on the type of irAE.

Because ir-IA tends to be chronic, we recommend earlier initiation of DMARD. Hydroxychloroquine, sulfasalazine, methotrexate (MTX), tumor necrosis factor inhibitors (TNFi), and interleukin 6 inhibitors have all been used successfully for inflammatory arthritis ${ }^{19,60,61}$. The choice of DMARD for ir-IA depends on severity of symptoms, patient preference, and access to medication. Mycophenolate mofetil (MMF) has been used preferentially for hepatitis, while TNFi are commonly used for colitis. Intravenous immunoglobulin, plasmapheresis, MTX, azathioprine, and MMF have been used for myositis ${ }^{39,62}$, and cyclophosphamide and plasmapheresis have been used for vasculitis ${ }^{44}$.

The decision to restart immunotherapy depends on type and severity of irAE, stage and response of underlying malignancy, and alternative treatment options, and should be done on a case-by-case basis with the patient and oncologist. In general, further immunotherapy is not recommended for those with Grade 3 or higher AE but may be necessary if there are no other options for underlying malignancy. One patient who developed dermatomyositis after ipilimumab was re-challenged with the same drug 14 months after discontinuation because of tumor recurrence. She had a flare of the dermatomyositis ${ }^{31}$. In contrast, 2 patients who developed ir-Myositis were re-challenged because of tumor progression, 1 with the same drug (avelumab) and the other with an alternative drug (ipilimumab/nivolumab replaced by pembrolizumab). Neither had a recurrence of myositis ${ }^{63}$.

\section{Effect of irAE treatment on cancer outcomes}

There are no prospective, randomized controlled trials evaluating whether concomitant immunosuppressive therapy negates the anti-tumor response of immunotherapy. Although most data suggest that the use of corticosteroid treatment is not associated with adverse tumor response ${ }^{64,65,66}$, recent reports have suggested that there may be an association with negative tumor response, particularly at higher doses ${ }^{62,67}$. No undesirable tumor effects have been reported with use of MMF, infliximab, or MTX ${ }^{16,65,66}$. These data come from small, retrospective studies, with potential for significant bias. Longterm data are needed, in larger populations, across various cancer types, and manifesting with different $\mathrm{AE}$.

Most guidelines recommend cessation of immunotherapy in patients with grade 2 or higher AE. This can lead to anxiety regarding progression of the underlying malignancy. Nevertheless, Schadendorf, et al found that despite discontinuing immunotherapy (nivolumab plus ipilimumab) because of adverse events, patients had longterm tumor responses similar to those who continued therapy ${ }^{68}$. Further, in a followup study, they found that patients whose cancer progressed after discontinuing immunotherapy were able to regain a favorable response with restarting treatment, with a similar toxicity profile ${ }^{69}$.
All currently published guidelines recommend cessation of immunotherapy with active $\mathrm{AE}$ requiring systemic immunosuppression. There is little experience with combination of longterm immunosuppression with ongoing immunotherapy. That said, our preference (similar to others in the literature ${ }^{22}$ ) is to continue immunotherapy in patients with inflammatory arthritis, because these respond to corticosteroids and disease-modifying drugs. More research is needed to understand the complex interactions and clinical implications involved in the longterm care of these patients.

A common clinical question is whether the development of irAE predicts a favorable tumor response. This would certainly make sense because it implies that immunotherapy has done its job in "turning on" the immune system to fight cancer, thus leading to an autoimmune reaction. The answer to this remains a topic of debate ${ }^{9}$. Many clinical studies have found a correlation between the development of irAE and favorable cancer outcomes ${ }^{66,70-76}$. This seems to be independent of tumor type (reported in melanoma, renal cell carcinoma, lung cancer), type of immunotherapy (observed for both PD-1 and CTLA-4 inhibitors), or type of adverse event (seen with endocrinopathies, vitiligo, colitis, arthritis, etc.). However, this finding has not been universal. A retrospective study of ipilimumab in 298 melanoma patients found no difference in tumor response between those who developed irAE and those who did not ${ }^{77}$. Further research is needed to conclusively answer this question.

\section{Preexisting rheumatic disease}

Patients with autoimmune disease (AID) were largely excluded from clinical trials, making cancer treatment decisions in these patients challenging ${ }^{78}$. A large systematic review of 123 patients with preexisting AID treated with ICI has been published ${ }^{79}$. Of these, 72 (59\%) had rheumatologic conditions including inflammatory arthritis, sarcoidosis, vasculitis, spondyloarthritis, Behçet disease, myositis, PMR, systemic lupus erythematosus, rheumatic fever, and Sjögren syndrome. Overall, about $50 \%$ had exacerbation of the preexisting AID and 34\% had de novo irAE. The occurrence of AE was not different between patients with active versus inactive preexisting AID, but fewer AE were seen in patients receiving therapy for their AID at the time of ICI initiation. Overall, $17.1 \%$ discontinued ICI permanently because of AE.

Additionally, Menzies and colleagues reported a retrospective review of 52 patients with preexisting AID, of which $27(52 \%)$ were classified as rheumatologic, who were treated with anti-PD-1 therapy for advanced melanoma ${ }^{80}$. The overall tumor response rate was 33\%. Thirty-eight percent had a flare of their underlying AID requiring immunosuppression, and only $4 \%$ discontinued ICI because of their AID flare. More recently, a study of 16 patients with preexisting rheumatic disease were treated with ICI. Of these, $6(38 \%)$ experienced an irAE, with only $1(6 \%)$ having a flare of the underlying rheumatologic disease ${ }^{81}$.

Personal non-commercial use only. The Journal of Rheumatology Copyright $\odot$ 2020. All rights reserved 
These reports show that ICI can be used successfully in patients with AID. The response rate and incidence of AE is still unclear and prospective studies are needed in patients with preexisting AID. Nevertheless, experts agree that having a preexisting AID is not an absolute contraindication for treatment with ICI. This challenging treatment decision should be made on a case-by-case basis with collaborative consultation among patient, oncologist, rheumatologist, and other specialists. Rheumatologists have an important role in the initial assessment of disease activity, treatment, and close monitoring of the underlying autoimmune disease. Further studies are needed to answer important questions such as effect of concomitant immunosuppressive therapy on tumor response, potential biomarkers to predict irAE, and optimal preventive and treatment strategies for patients with underlying autoimmune disease.

In the last decade, ICI have changed the model of cancer care. They now have an established role in all phases of cancer treatment, from (neo)adjuvant settings all the way to refractory metastatic disease, in an ever-growing number of indications. The enhancement of immune responses with ICI has led to the emergence of irAE. Much of the initial focus of irAE identification and management was on rash, colitis, and endocrinopathies, in part because these are more common and in part because they can be associated with significant morbidity.

To date, the importance and recognition of Rh-irAE has lagged. There are several reasons for this, including (1) many symptoms of Rh-irAE, including arthralgias and myalgias, can be mistakenly attributed to the underlying malignancy, (2) treating physicians may have difficulty recognizing synovitis or may underestimate the severity of the symptoms, (3) symptoms are often delayed, and (4) symptoms are unlikely to require hospitalization or be life-threatening. As awareness increases and experience accumulates, we are learning more about rheumatic irAE and their management. As experts in systemic autoimmune disease, rheumatologists are well suited to be important members of the team in identifying and treating patients with irAE and assisting with systemic immunosuppression.

There are also many outstanding questions and significant opportunities for education and research in the field of Rh-irAE. Thus, the Canadian Research Group of Rheumatology in Immuno-Oncology was created to share knowledge, develop rheumatology-specific management guidelines, and undertake studies to define the natural history and optimal treatment of Rh-irAE. The potential to compare and contrast Rh-irAE with traditional AID and to gain further insight into the latter is also an exciting prospect and bodes well for future collaborations with other established Canadian and international rheumatology cohorts.

\section{REFERENCES}

1. Khalil DN, Smith EL, Brentjens RJ, Wolchok JD. The future of cancer treatment: immunomodulation, CARs and combination immunotherapy. Nat Rev Clin Oncol 2016;13:394.

2. Horn L, Reck M, Gettinger SN, Spigel DR, Antonia SJ, Rupnow BA, et al. CheckMate 331: an open-label, randomized phase III trial of nivolumab versus chemotherapy in patients (pts) with relapsed small cell lung cancer (SCLC) after first-line platinum-based chemotherapy (PT-DC). J Clin Oncol 2016;34:15_suppl, TPS8578-TPS8578.

3. Topalian SL, Drake CG, Pardoll DM. Immune checkpoint blockade: a common denominator approach to cancer therapy. Cancer Cell 2015;27:450-61.

4. Postow MA, Callahan MK, Wolchok JD. Immune checkpoint blockade in cancer therapy. J Clin Oncol 2015;33:1974-82.

5. Dyck L, Mills KHG. Immune checkpoints and their inhibition in cancer and infectious diseases. Eur J Immunol 2017;47:765-79.

6. Buder-Bakhaya K, Benesova K, Schulz C, Anwar H, Dimitrakopoulou-Strauss A, Weber TF, et al. Characterization of arthralgia induced by PD-1 antibody treatment in patients with metastasized cutaneous malignancies. Cancer Immunol Immunother 2018;67:175-82.

7. Hargadon KM, Johnson CE, Williams CJ. Immune checkpoint blockade therapy for cancer: an overview of FDA-approved immune checkpoint inhibitors. Int Immunopharmacol 2018; 62:29-39.

8. Michot JM, Bigenwald C, Champiat S, Collins M, Carbonnel F, Postel-Vinay S, et al. Immune-related adverse events with immune checkpoint blockade: a comprehensive review. Eur J Cancer 2016;54:139-48.

9. Postow MA, Sidlow R, Hellmann MD. Immune-related adverse events associated with immune checkpoint blockade. N Engl J Med 2018;378:158-68.

10. Champiat S, Lambotte O, Barreau E, Belkhir R, Berdelou A, Carbonnel F, et al. Management of immune checkpoint blockade dysimmune toxicities: a collaborative position paper. Ann Oncol 2016;27:559-74.

11. Hofmann L, Forschner A, Loquai C, Goldinger SM, Zimmer L, Ugurel S, et al. Cutaneous, gastrointestinal, hepatic, endocrine, and renal side-effects of anti-PD-1 therapy. Eur J Cancer 2016;60: 190-209.

12. Larkin J, Chiarion-Sileni V, Gonzalez R, Grob JJ, Cowey CL, Lao $\mathrm{CD}$, et al. Combined nivolumab and ipilimumab or monotherapy in untreated melanoma. N Engl J Med 2015;373:23-34.

13. Day D, Hansen AR. Immune-related adverse events associated with immune checkpoint inhibitors. BioDrugs 2016;30:571-84.

14. Wang DY, Salem JE, Cohen JV, Chandra S, Menzer C, Ye F, et al. Fatal toxic effects associated with immune checkpoint inhibitors: a systematic review and meta-analysis. JAMA Oncol 2018;4:1721-8.

15. Cappelli LC, Shah AA, Bingham CO 3rd. Cancer immunotherapy-induced rheumatic diseases emerge as new clinical entities. RMD Open 2016;2:e000321.

16. Cappelli LC, Gutierrez AK, Bingham CO, Shah AA. Rheumatic and musculoskeletal immune-related adverse events due to immune checkpoint inhibitors: a systematic review of the literature. Arthritis Care Res 2017;69:1751-63.

17. Cappelli LC, Gutierrez AK, Baer AN, Albayda J, Manno RL, Haque $\mathrm{U}$, et al. Inflammatory arthritis and sicca syndrome induced by nivolumab and ipilimumab. Ann Rheum Dis 2017;76:43-50.

18. Kostine M, Rouxel L, Barnetche T, Veillon R, Martin F, Dutriaux C, et al. Rheumatic disorders associated with immune checkpoint inhibitors in patients with cancer-clinical aspects and relationship with tumour response: a single-centre prospective cohort study. Ann Rheum Dis 2018;77:393-8.

19. Moseley KF, Naidoo J, Bingham CO, Carducci MA, Forde PM, Gibney GT, et al. Immune-related adverse events with immune checkpoint inhibitors affecting the skeleton: a seminal case series. J Immunother Cancer 2018;6:104-12.

Personal non-commercial use only. The Journal of Rheumatology Copyright $\odot$ 2020. All rights reserved 
20. Lidar M, Giat E, Garelick D, Horowitz Y, Amital H, Steinberg-Silman Y, et al. Rheumatic manifestations among cancer patients treated with immune checkpoint inhibitors. Autoimmun Rev 2018;17:284-9.

21. Tocut M, Brenner R, Zandman-Goddard G. Autoimmune phenomena and disease in cancer patients treated with immune checkpoint inhibitors. Autoimmun Rev 2018;17:610-6.

22. Mooradian MJ, Nasrallah M, Gainor JF, Reynolds KL, Cohen JV, Lawrence DP, et al. Musculoskeletal rheumatic complications of immune checkpoint inhibitor therapy: a single center experience. Semin Arthritis Rheum 2019;48:1127-32.

23. Calabrese C, Kirchner E, Kontzias K, Velcheti V, Calabrese LH. Rheumatic immune-related adverse events of checkpoint therapy for cancer: case series of a new nosological entity. RMD Open 2017;3:e000412.

24. Ennis D, Jamal S, Hudson M, Ye C, Saltman A, Himmel M, et al. SAT0598 Rheumatic immune related adverse events associated with cancer immunotherapy: a nationwide multi-center Canadian cohort from the Canadian Research Group of Rheumatology in Immuno-oncology (CanRIO). Ann Rheum Dis 2019;78 Suppl 2:1393.

25. Cappelli LC, Brahmer JR, Forde PM, Le DT, Lipson EJ, Naidoo J, et al. Clinical presentation of immune checkpoint inhibitor-induced inflammatory arthritis differs by immunotherapy regimen. Semin Arthritis Rheum 2018:48:553-7.

26. Cappelli LC, Dorak MT, Bettinotti MP, Bingham CO, Shah AA. Association of HLA-DRB1 shared epitope alleles and immune checkpoint inhibitor-induced inflammatory arthritis. Rheumatology 2019;58:476-80.

27. Liewluck T, Kao JC, Mauermann ML. PD-1 inhibitor-associated myopathies: emerging immune-mediated myopathies. J Immunother 2018;41:208-11.

28. Kao JC, Liao B, Markovic SN, Klein CJ, Naddaf E, Staff NP, et al. Neurological complications associated with anti-programmed death 1 (PD-1) antibodies. JAMA Neurol 2017;74:1216-22.

29. Liu JZ. Association analyses identify 38 susceptibility loci for inflammatory bowel disease and highlight shared genetic risk across populations. Nat Genet 2015;47:979-86.

30. Bourgeois-Vionnet J, Joubert B, Bernard E, Sia MA, Pante V, Fabien N, et al. Nivolumab-induced myositis: A case report and a literature review. J Neurol Sci 2018;387:51-3.

31. Sheik Ali S, Goddard AL, Luke JJ, Donahue H, Todd DJ, Werchniak A, et al. Drug-associated dermatomyositis following ipilimumab therapy: a novel immune-mediated adverse event associated with cytotoxic T-lymphocyte antigen 4 blockade. JAMA Dermatol 2015;151:195-9.

32. Tauber M, Cohen R, Laly P, Josselin L, André T, Mekinian A. Severe necrotizing myositis associated with long term anti-neoplastic efficacy following nivolumab plus ipilimumab combination therapy. Clin Rheumatol 2019;38:601-2.

33. Uchio N, Taira K, Ikenaga C, Unuma A, Kadoya M, Kubota A, et al. Granulomatous myositis induced by anti-PD-1 monoclonal antibodies. Neurol Neuroimmunol Neuroinflammation 2018;5:e464.

34. Moslehi JJ, Salem JE, Sosman JA, Lebrun-Vignes B, Johnson DB. Increased reporting of fatal immune checkpoint inhibitor-associated myocarditis. Lancet 2018;391:933.

35. Suzuki S, Ishikawa N, Konoeda F, Seki N, Fukushima S, Takahashi $\mathrm{K}$, et al. Nivolumab-related myasthenia gravis with myositis and myocarditis in Japan. Neurology 2017;89:1127-34.

36. Suzuki S, Utsugisawa K, Yoshikawa H, Motomura M, Matsubara S, Yokoyama K, et al. Autoimmune targets of heart and skeletal muscles in myasthenia gravis. Arch Neurol 2009;66:1334-8.

37. Mammen AL, Rajan A, Pak K, Lehky T, Casciola-Rosen L, Donahue RN, et al. Pre-existing antiacetylcholine receptor autoantibodies and B cell lymphopaenia are associated with the development of myositis in patients with thymoma treated with avelumab, an immune checkpoint inhibitor targeting programmed death-ligand 1. Ann Rheum Dis 2019;78:150-2.

38. Shah M, Tayar JH, Abdel-Wahab N, Suarez-Almazor ME. Myositis as an adverse event of immune checkpoint blockade for cancer therapy. Semin Arthritis Rheum 2019;48:736-40.

39. Touat M, Maisonobe T, Knauss S, Ben Hadj Salem O, Hervier B, Auré K, et al. Immune checkpoint inhibitor-related myositis and myocarditis in patients with cancer. Neurology 2018;91:e985-94.

40. John S, Antonia SJ, Rose TA, Seifert RP, Centeno BA, Wagner AS et al. Progressive hypoventilation due to mixed CD8+ and CD4+ lymphocytic polymyositis following tremelimumab - durvalumab treatment. J Immunother Cancer 2017;5:54.

41. Anquetil C, Salem JE, Lebrun-Vignes B, Johnson DB, Mammen $\mathrm{AL}$, Stenzel W, et al. Immune checkpoint inhibitor-associated myositis. Circulation 2018;138:743-5.

42. Hunter G, Voll C, Robinson CA. Autoimmune inflammatory myopathy after treatment with ipilimumab. Can J Neurol Sci 2009;36:518-20.

43. Roberts JH, Smylie M, Oswald A, Cusnir I, Ye C. Hepatitis is the new myositis. Melanoma Res 2018;28:484-5.

44. Daxini A, Cronin K, Sreih AG. Vasculitis associated with immune checkpoint inhibitors - a systematic review. Clin Rheumatol 2018;37:2579-84.

45. Salem JE, Manouchehri A, Moey M, Lebrun-Vignes B, Bastarache L, Pariente A, et al. Cardiovascular toxicities associated with immune checkpoint inhibitors: an observational, retrospective, pharmacovigilance study. Lancet Oncol 2018;19:1579-89.

46. Comont T, Sibaud V, Mourey L, Cougoul P, Beyne-Rauzy O. Immune checkpoint inhibitor-related acral vasculitis. J Immunother Cancer 2018;6:120.

47. van den Brom RR, Abdulahad WH, Rutgers A, Kroesen BJ, Roozendaal C, de Groot DJ, et al. Rapid granulomatosis with polyangiitis induced by immune checkpoint inhibition. Rheumatology 2016;55:1143-5.

48. Minor DR, Bunker SR, Doyle J. Lymphocytic vasculitis of the uterus in a patient with melanoma receiving ipilimumab. J Clin Oncol 2013;31:e356.

49. Fierz F, Meier F, Chaloupka K, Böni C. Intraocular inflammation associated with new therapies for cutaneous melanoma - case series and review. Klin Monbl Augenheilkd 2016;233:540-4.

50. Manusow JS, Khoja L, Pesin N, Joshua AM, Mandelcorn ED. Retinal vasculitis and ocular vitreous metastasis following complete response to PD-1 inhibition in a patient with metastatic cutaneous melanoma. J Immunother Cancer 2014;2:41.

51. Castillo B, Gibbs J, Brohl AS, Seminario-Vidal L. Checkpoint inhibitor-associated cutaneous small vessel vasculitis. JAAD Case Reports 2018;4:675-7.

52. Maul LV, Weichenthal M, Kähler KC, Hauschild A. Successful anti-PD-1 antibody treatment in a metastatic melanoma patient with known severe autoimmune disease. J Immunother 2016;39:188-90.

53. Zhang H, Watanabe R, Berry GJ, Vaglio A, Liao YJ, Warrington KJ, et al. Immunoinhibitory checkpoint deficiency in medium and large vessel vasculitis. Proc Natl Acad Sci 2017;114:E970-9.

54. US Department of Health and Human Services. Common Terminology Criteria for Adverse Events (CTCAE) version 5.0. 2017. [Internet. Accessed October 31, 2019.] Available from: ctep.cancer.gov/protocolDevelopment/electronic_applications/docs/ CTCAE_v5_Quick_Reference_8.5x11.pdf

55. Brahmer JR, Lacchetti C, Schneider BJ, Atkins MB, Brassil KJ, Caterino JM, et al. Management of immune-related adverse events in patients treated with immune checkpoint inhibitor therapy: American Society of Clinical Oncology clinical practice guideline. J Clin Oncol 2018;36:1714-68.

56. Haanen JBAG, Carbonnel F, Robert C, Kerr KM, Peters S, Larkin J, 
et al; ESMO Guidelines Committee. Management of toxicities from immunotherapy: ESMO Clinical Practice Guidelines for diagnosis, treatment and follow-up. Ann Oncol 2017;28 Suppl 4:iv119-42.

57. Kumar V, Chaudhary N, Garg M, Floudas CS, Soni P, Chandra AB. Current diagnosis and management of immune related adverse events (irAEs) induced by immune checkpoint inhibitor therapy. Front Pharmacol 2017;8:49.

58. Puzanov I, Diab A, Abdallah K, Bingham CO, Brogdon C, Dadu R, et al. Managing toxicities associated with immune checkpoint inhibitors: consensus recommendations from the Society for Immunotherapy of Cancer (SITC) Toxicity Management Working Group. J Immunother Cancer 2017;5:95.

59. Cappelli LC, Shah AA, Bingham CO. Immune-related adverse effects of cancer immunotherapy - implications for rheumatology. Rheum Dis Clin North Am 2017;43:65-78.

60. Kim ST, Tayar J, Trinh VA, Suarez-Almazor M, Garcia S, Hwu P, et al. Successful treatment of arthritis induced by checkpoint inhibitors with tocilizumab: a case series. Ann Rheum Dis 2017;76:2061-4.

61. Calabrese LH, Calabrese C, Cappelli LC. Rheumatic immune-related adverse events from cancer immunotherapy. Nat Rev Rheumatol 2018;14:569-79.

62. Mitchell EL, Lau PKH, Khoo C, Liew D, Leung J, Liu B, et al. Rheumatic immune-related adverse events secondary to anti-programmed death-1 antibodies and preliminary analysis on the impact of corticosteroids on anti-tumour response: A case series. Eur J Cancer 2018;105:88-102.

63. Delyon J, Brunet-Possenti F, Leonard-Louis S, Arangalage D, Baudet M, Baroudjian B, et al; PATIO Group. Immune checkpoint inhibitor rechallenge in patients with immune-related myositis. Ann Rheum Dis 2019;78:e129.

64. Horvat TZ, Adel NG, Dang TO, Momtaz P, Postow MA, Callahan $\mathrm{MK}$, et al. Immune-related adverse events, need for systemic immunosuppression, and effects on survival and time to treatment failure in patients with melanoma treated with ipilimumab at Memorial Sloan Kettering Cancer Center. J Clin Oncol 2015;33:3193-8.

65. Beck KE, Blansfield JA, Tran KQ, Feldman AL, Hughes MS, Royal RE, et al. Enterocolitis in patients with cancer after antibody blockade of cytotoxic t-lymphocyte-associated antigen 4. J Clin Oncol 2006;24:2283-9.

66. Attia P, Phan GQ, Maker AV, Robinson MR, Quezado MM, Yang $\mathrm{JC}$, et al. Autoimmunity correlates with tumor regression in patients with metastatic melanoma treated with anti-cytotoxic T-lymphocyte antigen-4. J Clin Oncol 2005;23:6043-53.

67. Arbour KC, Mezquita L, Long N, Rizvi H, Auclin E, Ni A, et al. Impact of baseline steroids on efficacy of programmed cell death-1 and programmed death-ligand 1 blockade in patients with non-small-cell lung cancer. J Clin Oncol 2018;36:2872-8.

68. Schadendorf D, Wolchok JD, Hodi FS, Chiarion-Sileni V, Gonzalez R, Rutkowski P, et al. Efficacy and safety outcomes in patients with advanced melanoma who discontinued treatment with nivolumab and ipilimumab because of adverse events: a pooled analysis of randomized phase II and III trials. J Clin Oncol 2017;35:3807-14.

69. Robert C, Schadendorf D, Messina M, Hodi FS, O’Day S. Efficacy and safety of retreatment with ipilimumab in patients with pretreated advanced melanoma who progressed after initially achieving disease control. Clin Cancer Res 2013;19:2232-9.

70. Yang JC, Hughes M, Kammula U, Royal R, Sherry RM, Topalian SL, et al. Ipilimumab (anti-CTLA4 antibody) causes regression of metastatic renal cell cancer associated with enteritis and hypophysitis. J Immunother 2007;30:825-30

71. Downey SG, Klapper JA, Smith FO, Yang JC, Sherry RM, Royal $\mathrm{RE}$, et al. Prognostic factors related to clinical response in patients with metastatic melanoma treated by CTL-associated antigen-4 blockade. Clin Cancer Res 2007;13:6681-8.

72. Bouwhuis MG, Ten Hagen TL, Suciu S, Eggermont AM. Autoimmunity and treatment outcome in melanoma. Curr Opin Oncol 2011;23:170-6.

73. Hua C, Boussemart L, Mateus C, Routier E, Boutros C, Cazenave $\mathrm{H}$, et al. Association of vitiligo with tumor response in patients with metastatic melanoma treated with pembrolizumab. JAMA Dermatol 2016;152:45.

74. Shoushtari AN, Friedman CF, Navid-Azarbaijani P, Postow MA, Callahan MK, Momtaz P, et al. Measuring toxic effects and time to treatment failure for nivolumab plus ipilimumab in melanoma. JAMA Oncol 2018;4:98.

75. Haratani K, Hayashi H, Chiba Y, Kudo K, Yonesaka K, Kato R, et al. Association of immune-related adverse events with nivolumab efficacy in non-small-cell lung cancer. JAMA Oncol 2018;4:374.

76. Sato K, Akamatsu H, Murakami E, Sasaki S, Kanai K, Hayata A, et al. Correlation between immune-related adverse events and efficacy in non-small cell lung cancer treated with nivolumab. Lung Cancer 2018;115:71-4

77. Faje AT, Lawrence D, Flaherty K, Freedman C, Fadden R, Rubin K, et al. High-dose glucocorticoids for the treatment of ipilimumab-induced hypophysitis is associated with reduced survival in patients with melanoma. Cancer 2018;124:3706-14.

78. Johnson DB, Sullivan RJ, Menzies AM. Immune checkpoint inhibitors in challenging populations. Cancer 2017;123:1904-11.

79. Abdel-Wahab N, Shah M, Lopez-Olivo MA, Suarez-Almazor ME. Use of immune checkpoint inhibitors in the treatment of patients with cancer and preexisting autoimmune disease. Ann Intern Med 2018;168:121-30.

80. Menzies AM, Johnson DB, Ramanujam S, Atkinson VG, Wong ANM, Park JJ, et al. Anti-PD-1 therapy in patients with advanced melanoma and preexisting autoimmune disorders or major toxicity with ipilimumab. Ann Oncol 2017;28:368-76.

81. Richter MD, Pinkston O, Kottschade LA, Finnes HD, Markovic SN, Thanarajasingam $\mathrm{U}$. Brief report: cancer immunotherapy in patients with preexisting rheumatic disease: the Mayo Clinic experience. Arthritis Rheumatol 2018;70:356-60. 